\title{
$\mathrm{Hi}$ gh- speed at om c for ce mi croscopy shows dynam c mol ecul ar processes i n phot oact i vated bact er i or hodopsi $n$
}

\begin{tabular}{|l|l|}
\hline 著者 & $\begin{array}{l}\text { Shi bat a M ki hi ro, Yanashi ta Hay at o, Uchi hashi } \\
\text { Takayuki, Kandor i H deki, Ando Toshi o }\end{array}$ \\
\hline 著者別表示 & 柴田 幹大, 内橋 貴之, 安藤 敏夫 \\
\hline $\begin{array}{l}\text { j our nal or } \\
\text { publ i cat i on titl e }\end{array}$ & Nat ure Nanot echnol ogy \\
\hline vol une & 5 \\
\hline number & 3 \\
\hline page range & $208-212$ \\
\hline year & $2010-03-01$ \\
\hline URL & ht t p: //doi . or g/10. 24517/00011001 \\
\hline
\end{tabular}




\section{High-speed atomic force microscopy visualization reveals dynamic molecular processes in photo-activated bacteriorhodopsin}

Mikihiro Shibata ${ }^{1}$, Hayato Yamashita ${ }^{1}$, Takayuki Uchihashi ${ }^{1,2}$, Hideki Kandori ${ }^{3} \&$ Toshio Ando ${ }^{1,2}$

${ }^{1}$ Department of Physics, Kanazawa University, Kakuma-machi, Kanazawa 920-1192, Japan. ${ }^{2}$ Core Research for Evolutional Science and Technology (CREST) of the Japan Science and Technology Agency (JST), Sanban-cho, Chiyoda-ku, Tokyo 102-0075, Japan. ${ }^{3}$ Department of Frontier Materials, Nagoya Institute of Technology, Showa-ku, Nagoya 466-8555, Japan.

Dynamic changes in protein conformation in response to external stimuli are vital in biological processes. Their direct visualization under physiological conditions has long been desired, but remains a challenge. Here, we demonstrate that high-speed atomic force microscopy, which has recently been developed by us, now makes such visualization feasible. The high-resolution movies of a light-driven proton pump, bacteriorhodopsin (bR), reveal that upon illumination, a cytoplasmic portion of each bR monomer is brought into contact with the adjacent trimers. Remarkably, the bR-bR interaction in the transiently formed assembly elicits both positive and negative cooperative effects on the decay kinetics as the initial bR recovers. By the bipolar nature of the cooperativity, however, the turnover rate of the phtocycle is maintained constant on average, irrespective of the light intensity. Thus, the direct and high-resolution visualization of dynamically acting molecules is a powerful new approach to gaining insight into elaborate bimolecular processes. 
The biological function of proteins is closely associated with their ability to undergo structural changes. In many cases, these structural changes are triggered by external stimuli including $\mathrm{pH}$, temperature, ligand binding, mechanical stress, and light. Although their direct real-space and real-time visualization is a straightforward approach to understanding the dynamic molecular processes, the lack of suitable techniques has precluded it. Atomic force microscopy (AFM) is a versatile technique to image proteins in liquids at sub-molecular resolution, but its poor temporal resolution has meant an availability of only static or slow time-lapse images of proteins ${ }^{1-5}$. In the last decade, various efforts have been carried out to increase the scan speed of $\mathrm{AFM}^{6-9}$. As a result, single protein molecules exhibiting Brownian motion are captured on video at a highest temporal resolution of $\sim 30 \mathrm{~ms}^{10}$. However, dynamic visualization of physiologically relevant conformational changes in proteins has been difficult because tip-sample interaction tends to interfere with the physiological functions. To solve this problem, a new method has recently been developed which allows fast and precise control of the tip-sample distance with a minimum load to the sample ${ }^{7}$ This report presents the first ever exemplification of dynamic imaging of a functioning biological sample.

Bacteriorhodopsin (bR) is a well-known example of the association between stimulus-triggered structural dynamics and biological function ${ }^{11,12}$, and its direct visualization has long been a goal. bR contains seven transmembrane $\alpha$-helices (named A-G) enclosing the chromophore retinal ${ }^{13,14}$. In the photocycle, a series of spectral intermediates, designated $\mathrm{J}, \mathrm{K}, \mathrm{L}, \mathrm{M}, \mathrm{N}$, and $\mathrm{O}$, occur in that $\operatorname{order}^{12}$. The light-induced conformational changes in $\mathrm{bR}$ have been investigated by various methods ${ }^{15-25}$, leading to a consensus that the proton channel at the cytoplasmic surface is opened by the tilting of helix F away from the protein center ${ }^{21,23,24}$. Sass et al. reported helix F displacement of $\sim 0.1 \mathrm{~nm}$ in the late $\mathrm{M}$ state, based on X-ray diffraction of the three-dimensional crystal of wild type $(\mathrm{WT})^{21}$. However, a larger structural change in bR was reported in 
the electron crystallography study of the D96G, F171C, F219L triple mutant of bR: displacement of helix $\mathrm{F}$ by $\sim 0.35 \mathrm{~nm}$ away from the center of the protein ${ }^{23}$. The electron crystallography study of the F219L mutant further reported that helices E and F tilt away from the center of the protein, which is followed by a shift of the E-F loop by $\sim 0.3$ $\mathrm{nm}^{24}$, resulting in large-scale conformational changes in the $\mathrm{M}$ and $\mathrm{N}$ states ${ }^{18,19,25}$. Nevertheless, a consensus on the conformational changes is not reached yet. Similarly to other proteins, our knowledge of the structural dynamics of bR is limited because its structural data have been accumulated using static and ensemble averaging approaches. The direct and dynamic visualization by high-speed AFM will provide a better answer as to the conformational changes of $b R$.

We used the D96N bR mutant, which has a longer photocycle $(\sim 10 \mathrm{~s})$ than that of WT $(\sim 10 \mathrm{~ms})$ but retains proton pumping ability ${ }^{26}$. Figure 1a shows successive images of D96N at the cytoplasmic surface captured at 1 frame/s (fps) (Supplementary Movie 1 online). Individual bR molecules are clearly seen, as reported in earlier pioneering studies for the unphotolyzed state ${ }^{3-5}$. Upon illumination with green light, bR drastically changes its structure (compare images at $1 \mathrm{~s}$ and $2 \mathrm{~s}$ ) and returns to the unphotolyzed state in a few seconds after light-off, an outcome that is reproducible in repeated dark-illumination cycles (Supplementary Movie 1 online). Such high-resolution movies of the dynamically changing structure of stimulated proteins have never been seen before.

We analyzed the "mass center" positions of the individual monomers imaged during the dark-illumination cycles. Note that the "mass center" for each bR monomer was calculated from the corresponding surface area and height distribution in the image (see Methods). All the activated bR monomers exhibited displacements of their center of mass and the average displacement was $0.69 \pm 0.15 \mathrm{~nm}$ (Supplementary Fig. 1 in the green region). In Figure 1b, the trajectories of the mass-center positions during the dark-illumination cycles are superimposed on the AFM image in the unphotolyzed state 
(red, blue, and black marks). They show highly centrosymmetric trajectories, oriented outward from the trimer centers.

The top and bottom images in Figure 1c show magnified images obtained before and during illumination, respectively. The prominent protrusion in the AFM topographs reflects interhelical loop structures on the cytoplasmic surface ${ }^{4,27}$. Compared to the atomic model of the $\alpha$-helical cytoplasmic ends in the unphotolyzed state ${ }^{14}$ (A-G in Fig. 1b and c), the protruding areas around helices $E$ and $F$ shift outward from the trimer center under illumination. Therefore, the protrusion movement visualized here is ascribed to the displacement of the E-F loop. As a result of E-F loop displacement, the protrusion is divided into two sections (green arrows 1 and 2 in Fig. 1c), and the minor protrusion (green arrow 2) likely corresponds to the position around helices A and B. Note that the overall position of each bR molecule does not change because of indiscernible alterations at the extracellular surface (Supplementary Fig. 2 and Movie 5 online).

To ensure that the conformational changes observed here are not artifacts such as tip-force-induced structural alterations ${ }^{4}$, we measured the decay of the active state after flash illumination at various $\mathrm{pH}$ values (Fig. 1d). UV-visible spectroscopy ${ }^{26}$ has shown that the lifetime of the M-intermediate of D96N and WT is prolonged at alkaline $\mathrm{pH}$; in concordance with this fact is the observation here with D96N that the decay detected by high-speed AFM showed a strong $\mathrm{pH}$ dependence (inset, Fig. 1d). Using $\mathrm{WT}$ at $\mathrm{pH} 7$ and $\mathrm{pH}$ 10, this finding was further confirmed (Fig. 2, Supplementary Movies 2 and 3 online). Because of the very fast photocycle, no conformational changes of WT were detected at $\mathrm{pH} 7$, whereas at $\mathrm{pH} 10$, conformational changes similar to those of $\mathrm{D} 96 \mathrm{~N}$ were observed, although the activated state decays much faster than that of D96N.

The activation of D96N was observed within $1 \mathrm{~s}$ immediately after illumination but not observed after light-off, even at alkaline $\mathrm{pH}$ (data not shown). A cysteine accessibility study with D96N indicated displacement of the E-F loop in the M state ${ }^{19}$. 
Therefore, the high-speed AFM imaging of D96N did capture the light-induced ground-to-M-state transition. Since the AFM observation was performed with the samples on a mica surface, we cannot exclude a possible effect of the surface. Despite this uncertainty, the longer decay constant observed using AFM than that observed optically suggests that the activated state detected by AFM may contain an additional state in which the Schiff base is reprotonated (during $M$ decay) but the protein conformation has not yet returned to the ground state.

As a result of the outward displacement of the E-F loop, nearest-neighbor bR monomers, each belonging to a different adjacent trimer, transiently assemble. Here, we use a new designation, "trefoil", for the triad of the nearest-neighbor monomers to distinguish it from the original trimer. Remarkably, this transient assembly in a trefoil alters the decay kinetics of the activated state (Supplementary Movies 4 online). Figure $3 \mathrm{a}$ shows the time course of the displacement of the mass centers for six bR monomers belonging to two different trefoils observed at different light intensities $\left(\mathrm{M}_{\mathrm{n}} 1-\mathrm{M}_{\mathrm{n}} 3 ; \mathrm{n}\right.$ indicates different trefoils) (Supplementary Fig. 3 online). Under weak illumination, mostly only one monomer in each trefoil is activated $\left(\mathrm{M}_{1} 3\right.$ and $\mathrm{M}_{2} 2$ in the left panel of Fig. 3a). When only one monomer is activated in a trefoil, it decays with a time constant of $7.3 \pm 0.58 \mathrm{~s}$ (the top panel of Fig. 3b). In contrast, under stronger illumination, two or three monomers within a trefoil are tends to be activated together. Interestingly, the decay of each monomer markedly depends on the order of its activation. The monomer that is activated latest among the activated monomers in the trefoil (blue arrows in the right panel of Fig. 3a) decay with a shorter time constant of $2.0 \pm 0.16 \mathrm{~s}$ (the middle panel of Fig. 3b). On the other hand, the decay kinetics of the early activated monomers $\left(\mathrm{M}_{1} 2\right.$ and $\mathrm{M}_{2} 2$ in the right panel of Fig. 3a) does not follow a single exponential (the bottom panel of Fig. 3b), and the averaged decay time lengthens to about $13 \mathrm{~s}$. This observation indicates that the early activated monomers do not return to the ground state as long as the adjacent monomers within the trefoil are in the active 
state.

In addition, Figure 4 shows decay of the activated D96N under weak or strong light intensity. Note that all the activated molecules were counted regardless of their activation order and of the number of activated monomers in a trefoil. The distributions of the activated state lifetime are well fit to single exponential functions under both weak and strong light illumination, and their decay constants are almost identical despite of the different light intensities. This can be explained by the coexistence of the positive and negative cooperativity effects elicited by bR-bR interactions within a trefoil (Fig. 3b). Because of the coexistence and the bipolar nature, the two cooperative effects cancel out with each other on averaging, resulting in a constant decay rate independent of light intensity. Under a conceivable assumption that the conformational change turnover of an activated bR monmer is tightly coupled with proton transfer by the $\mathrm{bR}$ monomer, the average rate of proton-pumping by the activated bR would be conserved independently of light intensity. As a result of the cancelation effect, the cooperative effects can never be detected by ensemble averaging approaches. Thus, the cooperative effects are different from those proposed from absorption spectroscopic studies in which fast and slow decays of the M-state were detected depending on light intensity while the total turnover rate was independent of light intensity ${ }^{28,29}$. This previous report is consistent with our presumption that the activated state detected by high-speed AFM contains both the M-state and subsequent states. Importantly, the cooperative effects we found are caused by $\mathrm{bR}-\mathrm{bR}$ interactions within a trefoil, not within a trimer.

As demonstrated above, direct and real-space visualization of the dynamically changing structure of stimulated proteins provides a straightforward way of elucidating how protein molecules function. We can expect that a large number of dynamic bimolecular processes will be directly visualized in the near future, leading to new findings inaccessible using other approaches. After further advances of high-speed AFM techniques, even molecular processes on membranes of live cells will be directly 
visualized. The present study is the first step toward achieving these expected innovations in biological research.

\section{METHODS}

Sample preparation. Purple membranes containing either WT or D96N mutant were isolated from Halobacterium salinarum as described ${ }^{30}$. The samples were suspended in a solution containing $10 \mathrm{mM}$ Tris- $\mathrm{HCl}(\mathrm{pH} 7,8,9$, or 10) and $300 \mathrm{mM} \mathrm{KCl}$.

High-speed AFM observation. The laboratory-built high-speed AFM apparatus is an extensively improved version of the previously reported one ${ }^{6}$. See a comprehensive review $^{7}$ for the instrument development including recent technical advances. AFM images were acquired in the tapping mode. To detect the cantilever deflection, we used an optical beam deflection detector equipped with an infrared laser $(980 \mathrm{~nm})$. The laser beam was focused onto a small cantilever using a $\times 50$ objective lens. The cantilever (Olympus) is 6-7 $\mu \mathrm{m}$ long, $2 \mu \mathrm{m}$ wide, and $90 \mathrm{~nm}$ thick with a spring constant of $0.1-0.2 \mathrm{~N} / \mathrm{m}$. Its resonant frequency and quality factor in an aqueous solution are $\sim 1$ $\mathrm{MHz}$ and $\sim 2$, respectively. In the AFM imaging, the free oscillation amplitude was $\sim 1$ $\mathrm{nm}$ and the set-point amplitude was $90 \%$ of the free amplitude. The tapping force estimated was less than $30 \mathrm{pN}$. An amorphous carbon tip was grown on the original tip by electron beam deposition. The tip length was adjusted to $\sim 1 \mu \mathrm{m}$, and the tip apex was sharpened by plasma etching under argon gas ( $\sim 4 \mathrm{~nm}$ in radius). AFM observations were performed under solution at room temperature. For illumination of the bR sample, a green laser $(532 \mathrm{~nm})$ was irradiated through the $\times 50$ objective lens. The intensity measured at the exit of the objective lens was varied in a range of $0.004-0.5 \mu \mathrm{W}$.

Data analysis of mass-center position. The mass-center positions of imaged bR monomers were calculated by the following procedures. First, a whole AFM image was segmented into each monomer region by a watershed algorithm. Second, the grayscale 
of each segment was digitized using a threshold determined by a fuzzy-entropy algorithm. The pixels with grayscales above the threshold were defined as those of a monomer (region of interest; ROI). Finally the center of mass of a monomer was calculated for the ROI using the pixel positions and the grayscales. Note that the center of mass analyzed here is not the real center of mass of the protein but of the AFM image of the monomer.

\section{REFERENCES}

1. Binnig, G., Quate, C.F. \& Gerber, C. Atomic force microscope. Phys. Rev. Lett. 56, 930-933 (1986).

2. Drake, B. et al. Imaging crystals, polymers, and processes in water with the atomic force microscope. Science 243, 1586-1589 (1989).

3. Butt, H.J., Downing, K.H. \& Hansma, P.K. Imaging the membrane protein bacteriorhodopsin with the atomic force microscope. Biophys. J. 58, 1473-1480 (1990).

4. Müller, D.J. et al. Atomic force microscopy of native purple membrane. Biochim. Biophys. Acta 1460, 27-38 (2000).

5. Müller, D.J. AFM: a nanotool in membrane biology. Biochemistry 47, 7986-7998 (2008).

6. Ando, T. et al. A high-speed atomic force microscope for studying biological macromolecules. Proc. Natl. Acad. Sci. USA 98, 12468-12472 (2001).

7. Ando, T., Uchihashi, T., \& Fukuma, T. High-speed atomic force microscopy for nano-visualization of dynamic biomolecular processes. Prog. Sur. Sci. 83, 337-437 (2008).

8. Hansma, P.K., Schitter, G., Fantner, G.E. \& Prater, C. Applied physics. High-speed atomic force microscopy. Science 314, 601-602 (2006).

9. Fantner, G.E. et al. Components for high speed atomic force microscopy. 
Ultramicroscopy 106, 881-887 (2006).

10. Yamashita, H. et al. Tip-sample distance control using photothermal actuation of a small cantilever for high-speed atomic force microscopy. Rev. Sci. Instrum. 78, 083702 (2007).

11. Haupts, U., Tittor, J. \& Oesterhelt, D. Closing in on bacteriorhodopsin: progress in understanding the molecule. Annu. Rev. Biophys. Biomol. Struct. 28, 367-399 (1999).

12. Lanyi, J.K. Bacteriorhodopsin. Annu. Rev. Physiol. 66, 665-688 (2004).

13. Kimura, Y. et al. Surface of bacteriorhodopsin revealed by high-resolution electron crystallography. Nature 389, 206-211 (1997).

14. Luecke, H., Schobert, B., Richter, H.T., Cartailler, J.P. \& Lanyi, J.K. Structure of bacteriorhodopsin at 1.55 Å resolution. J. Mol. Biol. 291, 899-911 (1999).

15. Dencher, N.A., Dresselhaus, D., Zaccai, G. \& Buldt, G. Structural changes in bacteriorhodopsin during proton translocation revealed by neutron diffraction. Proc. Natl. Acad. Sci. USA 86, 7876-7879 (1989).

16. Subramaniam, S., Gerstein, M., Oesterhelt, D. \& Henderson, R. Electron diffraction analysis of structural changes in the photocycle of bacteriorhodopsin. EMBO J. 12, 1-8 (1993).

17. Kamikubo, H. et al. Structure of the $\mathrm{N}$ intermediate of bacteriorhodopsin revealed by x-ray diffraction. Proc. Natl. Acad. Sci. USA 93, 1386-1390 (1996).

18. Thorgeirsson, T.E. et al. Transient channel-opening in bacteriorhodopsin: an EPR study. J. Mol. Biol. 273, 951-957 (1997).

19. Brown, L.S., Needleman, R. \& Lanyi, J.K. Conformational change of the E-F interhelical loop in the M photointermediate of bacteriorhodopsin. J. Mol. Biol. 317, 471-478 (2002).

20. Shibata, M. \& Kandori, H. FTIR studies of internal water molecules in the Schiff base region of bacteriorhodopsin. Biochemistry 44, 7406-7413 (2005). 
21. Sass, H.J. et al. Structural alterations for proton translocation in the M state of wild-type bacteriorhodopsin. Nature 406, 649-653 (2000).

22. Luecke, H., Schobert, B., Richter, H.T., Cartailler, J.P. \& Lanyi, J.K. Structural changes in bacteriorhodopsin during ion transport at 2 angstrom resolution. Science 286, 255-261 (1999).

23. Subramaniam, S. \& Henderson, R. Molecular mechanism of vectorial proton translocation by bacteriorhodopsin. Nature 406, 653-657 (2000).

24. Vonck, J. Structure of the bacteriorhodopsin mutant F219L N intermediate revealed by electron crystallography. EMBO J. 19, 2152-2160 (2000).

25. Xiao, W., Brown, L.S., Needleman, R., Lanyi, J.K. \& Shin, Y.K. Light-induced rotation of a transmembrane alpha-helix in bacteriorhodopsin. J. Mol. Biol. 304, 715-721 (2000).

26. Otto, H. et al. Aspartic acid-96 is the internal proton donor in the reprotonation of the Schiff base of bacteriorhodopsin. Proc. Natl. Acad. Sci. USA 86, 9228-9232 (1989).

27. Heymann, J.B. et al. Charting the surfaces of the purple membrane. J. Struct. Biol. 128, 243-249 (1999).

28. Korenstein, R., Hess, B. \& Markus, M. Cooperativity in the photocycle of purple membrane of Halobacterium halobium with a mechanism of free energy transduction. FEBS Lett. 102, 155-161 (1979).

29. Varo, G., Needleman, R. \& Lanyi, J.K. Protein structural change at the cytoplasmic surface as the cause of cooperativity in the bacteriorhodopsin photocycle. Biophys. J. 70, 461-467 (1996).

30. Oesterhelt, D. \& Stoeckenius, W. Isolation of the cell membrane of Halobacterium halobium and its fractionation into red and purple membrane. . Methods Enzymol. 31, 667-678 (1973).

Note: Supplementary information is available on the Nature Nanotechnology website. 


\section{ACKNOWLEDGEMENTS}

This work was supported by JST CREST (T.A.), Grants-in-Aids for Scientific Research from JSPS (No. 15101005, T.A.; No. 19042009, T.U.; No. 20108014, H.K.) and Research Fellowships of JSPS for Young Scientists (M.S. and H.Y.). We thank J. K. Lanyi, S. P. Balashov, and L. S. Brown for comments on the draft.

Correspondence and requests for materials should be addressed to $\mathrm{T}$. A. (tando@kenroku.kanazawa-u.ac.jp) 


\section{FIGURE LEGENDS}

Figure 1 High-speed AFM images of the cytoplasmic surface of D96N under dark or illuminated conditions. (a) Successive AFM images of D96N bR adsorbed onto a mica surface in $10 \mathrm{mM}$ Tris-HCl $(\mathrm{pH} 7)$ and $300 \mathrm{mM} \mathrm{KCl}$. Frame rate: 1 fps; pixel size: $200 \times 200$ pixels (Supplementary Movie 1 online). A bR trimer is highlighted by the white triangle. The green bars indicate illumination of 532-nm green light with $0.5 \mu \mathrm{W}$. (b) Traces (red, blue, and black marks) of the mass-center positions under the dark-illumination cycles are superimposed on the image of D96N in the dark. The photo-induced movement of bR includes counterclockwise rotation $\left(7.4 \pm 2.2^{\circ}\right)$ around the trimer center. (c) Surface maps of the magnified images in the dark (upper panel) and under illumination (bottom panel). The position of each trimer center is denoted by the white dots. A monomer in the dark is indicated by the single white arrow. Under illumination, the topography of the monomer splits into major and minor protrusions as indicated by the green arrows 1 and 2, respectively. (d) Decay after flash-illumination of the activated state at different $\mathrm{pH}$ values $(7,8$, or 9$)$ detected by high-speed AFM. The exponential decay constants $(\tau)$ at $\mathrm{pH} 7,8$, and 9 are $6.7 \pm 0.10 \mathrm{~s}$ (the total number of analyzed bR molecules, $n_{\mathrm{T}}=320$; the number of different molecules analyzed, $n_{\mathrm{m}}=52$ ), $25 \pm 0.25 \mathrm{~s}\left(n_{\mathrm{T}}=373, n_{\mathrm{m}}=65\right)$, and $48 \pm 0.59 \mathrm{~s}\left(n_{\mathrm{T}}=214, n_{\mathrm{m}}=120\right)$, respectively. The inset shows the absorbance change at $410 \mathrm{~nm}$ (i.e., decay of the M-intermediate) after flash-illumination of D96N measured at various $\mathrm{pH}(7,8$, or 9). The exponential decay constants $(\tau)$ at $\mathrm{pH} 7,8$, and 9 are $3.4 \pm 0.025 \mathrm{~s}, 14 \pm 0.034 \mathrm{~s}$, and $33 \pm 0.080 \mathrm{~s}$, respectively.

Figure 2 Displacement of mass-center positions for WT at the cytoplasmic surface. Typical 10 bR monomers at pH 7 (a) and pH 10 (b) are analyzed. The AFM movies for WT at pH 7 and pH 10 are shown in Supplementary Movies 2 and 3 online, respectively. The green regions show periods of green light illumination (532 nm, 0.5 $\mu \mathrm{W})$. Conformational changes in WT are not detected at $\mathrm{pH} 7$ because the photocycle is 
too fast, whereas at $\mathrm{pH} 10$, they were repeatedly detected because the photocycle is prolonged at alkaline $\mathrm{pH}^{29}$.

Figure 3 Cooperative effects on the decay kinetics in D96N. (a) Displacement of mass-center positions as a function of time measured for six bR monomers composing two different trefoils $\left(\mathrm{M}_{\mathrm{n}} 1-\mathrm{M}_{\mathrm{n}} 3 ; \mathrm{n}\right.$ indicates different trefoils) at $\mathrm{pH} 7$ under different light intensities (Supplementary Fig. 3 and Movie 4 online). The green regions correspond to the illumination, and their shade level indicates the relative light intensity $(0.007 \mu \mathrm{W}$ and $0.5 \mu \mathrm{W})$. The blue arrows indicate the conformational changes of the monomers $\left(\mathrm{M}_{1} 3, \mathrm{M}_{2} 1\right.$, and $\left.\mathrm{M}_{2} 3\right)$ that are activated subsequently to the activation of other monomers in the respective trefoils. (b) The top figure shows the decay of activated bR monomers (as depicted by the red circle) while the other monomers (the gray circles) within the respective trefoils are not in the activated state. The exponential decay constant $(\tau)$ is $7.3 \pm 0.58 \mathrm{~s}\left(n_{\mathrm{T}}=279, n_{\mathrm{m}}=55\right)$. On the other hand, when one or two nearest neighbor molecules (as depicted by green circles in the middle figure) in a trefoil are already activated, the bR monomer activated latest (as depicted by the blue circles in the middle figure) decays faster. The exponential time constant $(\tau)$ is shortened to $2.0 \pm 0.16 \mathrm{~s}\left(n_{\mathrm{T}}=228, n_{\mathrm{m}}=55\right)$. In contrast, the early activated molecules within a trefoil (as depicted by green circles in the bottom figure) show a non-exponential broad distribution of decay time with an average of $13.3 \mathrm{~s}\left(n_{\mathrm{T}}=194\right.$, $\left.n_{\mathrm{m}}=55\right)$.

Figure 4 Decay rate of activated D96N under different light intensities. The upper figure shows the decay of activated molecules under weak illumination (0.004-0.09 $\mu \mathrm{W})$. Although the frequency is low, two bR monomers in a trefoil are activated together, the events of which are also counted in this analysis. The exponential decay constant $(\tau)$ is $5.4 \pm 0.34 \mathrm{~s}\left(n_{\mathrm{T}}=429, n_{\mathrm{m}}=55\right)$. The bottom figure shows the decay of 
activated molecules under relatively strong illumination $(0.23$ or $0.5 \mu \mathrm{W})$. The exponential decay constant $(\tau)$ is $6.1 \pm 0.37 \mathrm{~s}\left(n_{\mathrm{T}}=416, n_{\mathrm{m}}=55\right)$. 
Figure-1(Ando)

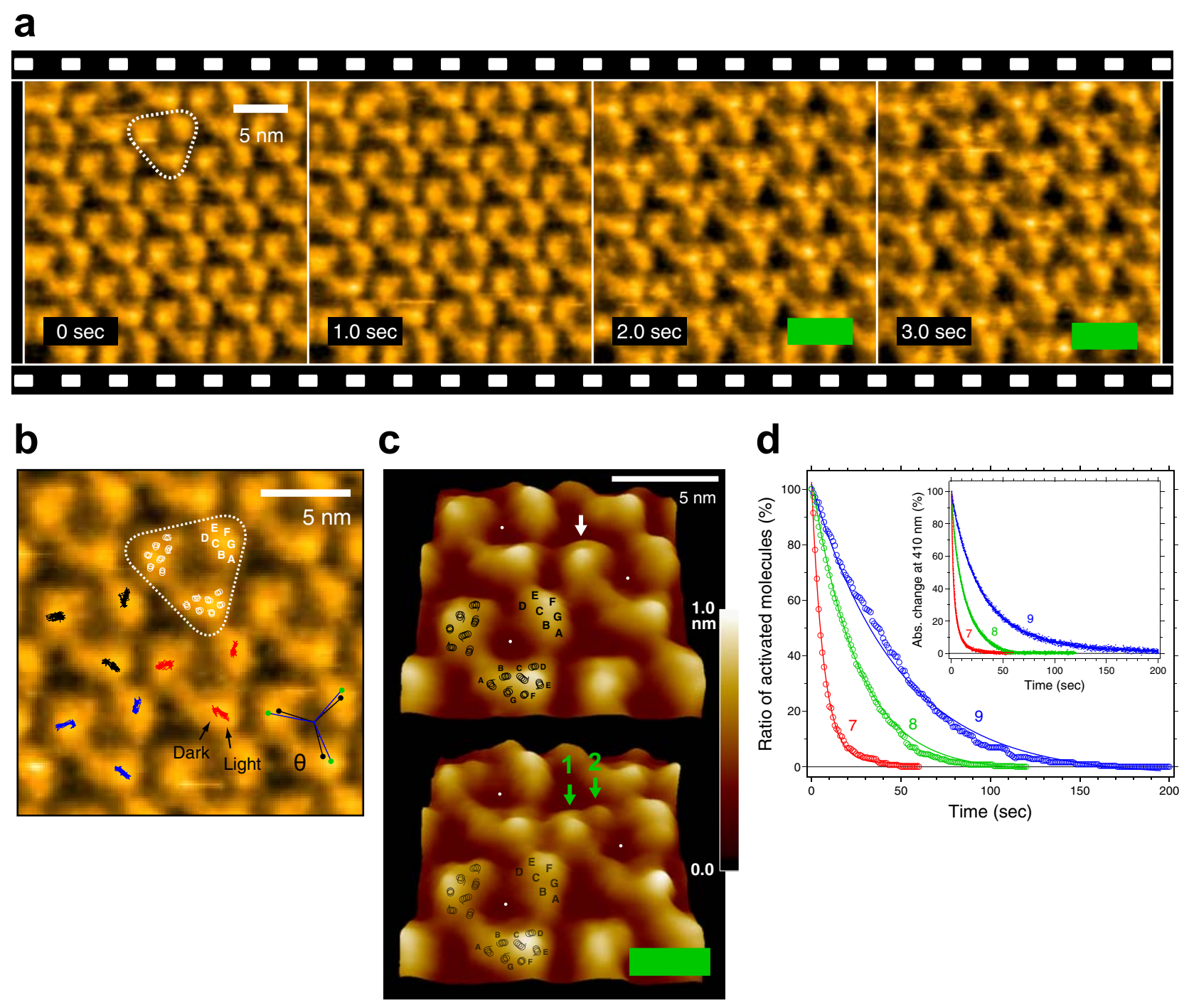




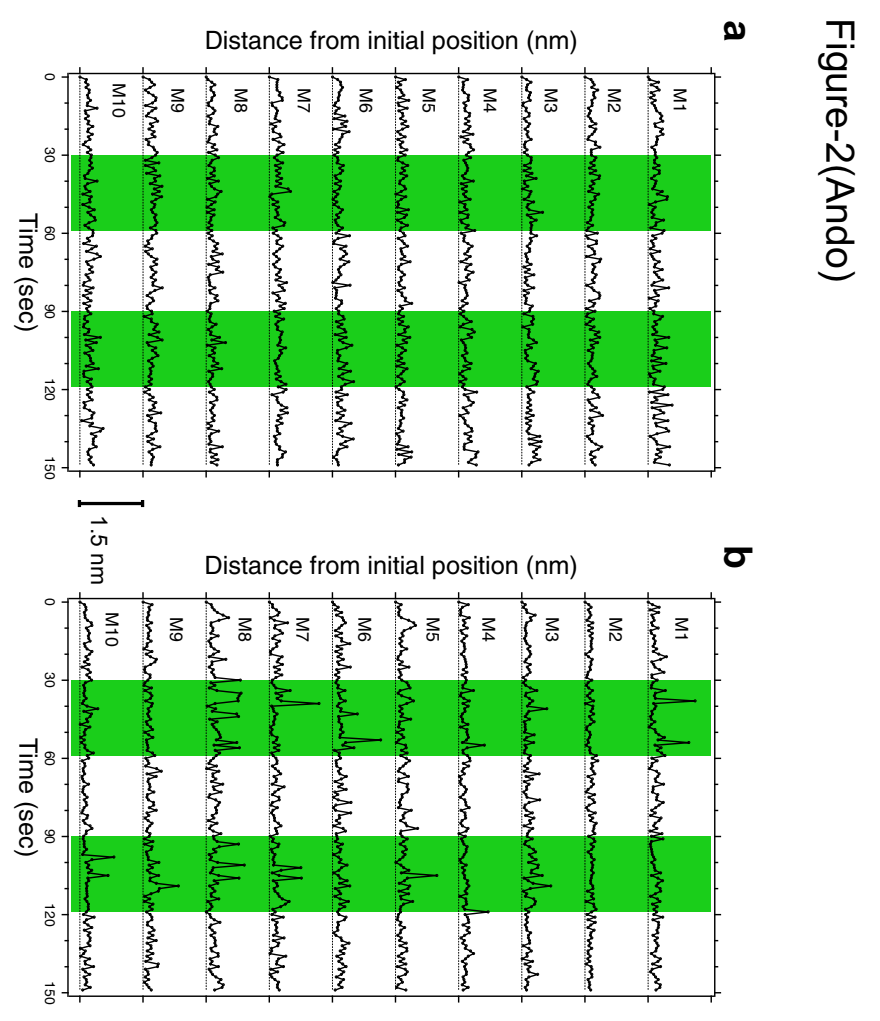


Figure-3(Ando)
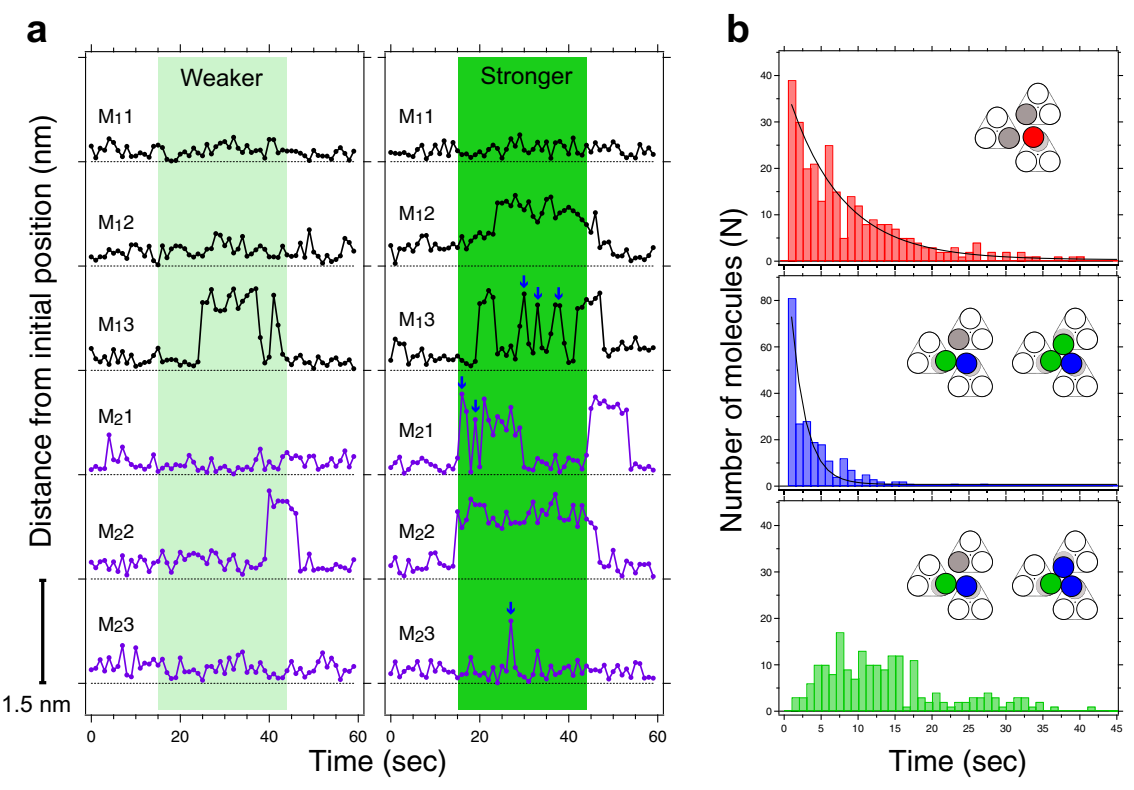
Figure-4(Ando)

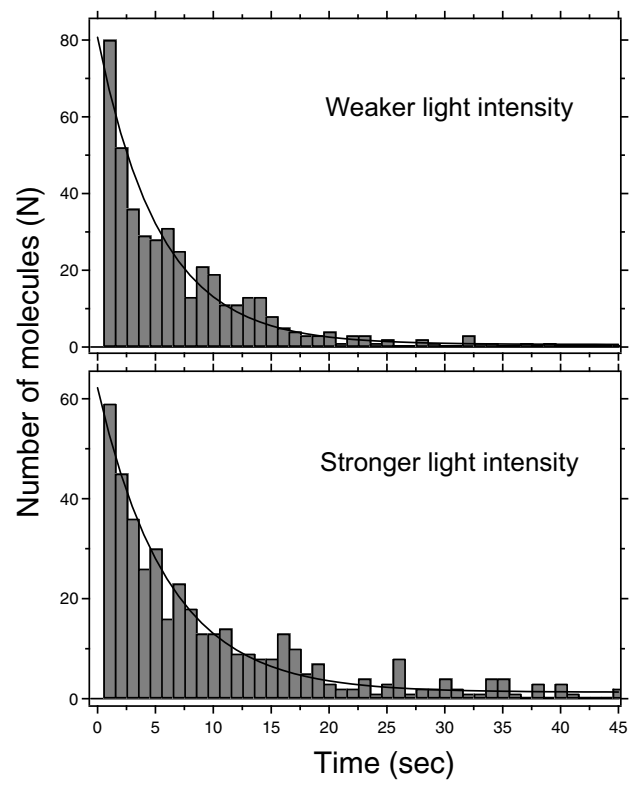

